\title{
Global Stability of Non-monotone Noncritical Traveling Waves for a Discrete Diffusion Equation with a Convolution Type Nonlinearity
}

\author{
Tao Su and Guo-Bao Zhang*
}

\begin{abstract}
This paper is concerned with the global stability of non-monotone traveling waves for a discrete diffusion equation with a monostable convolution type nonlinearity. It has been proved by Yang and Zhang (Sci. China Math. 61 (2018), 1789-1806) that all noncritical traveling waves (waves with speeds $c>c_{*}, c_{*}$ is minimal speed) are time-exponentially stable, when the initial perturbations around the waves are small. In this paper, we further prove that all traveling waves with large speed are globally stable, when the initial perturbations around the waves in a weighted Sobolev space can be arbitrarily large. The approaches adopted are the nonlinear Halanay's inequality, the technical weighted energy method and Fourier's transform.
\end{abstract}

\section{Introduction}

In this paper, we study the following spatially discrete diffusion equation with convolution type nonlinearity 8,28 :

$$
\frac{\partial u(t, x)}{\partial t}=\mathcal{D}_{2}[u](t, x)-u(t, x)+\sum_{i \in \mathbb{Z}} K(i) g(u(t-\tau, x-i)), \quad t>0, x \in \mathbb{R}
$$

with the initial data

$$
u(s, x)=u_{0}(s, x), \quad s \in[-\tau, 0], x \in \mathbb{R},
$$

where $\tau>0$ and

$$
\mathcal{D}_{2}[u](t, x)=d[u(t, x+1)-2 u(t, x)+u(t, x-1)] .
$$

Equation (1.1) models the matured population dynamics of a single species with nonzero maturation delay. Here, $u(t, x)$ represents the mature population at the time $t$ and the location $x, d>0$ is the coefficient of spacial diffusion, $\tau$ is the maturation delay,

Received January 18, 2019; Accepted September 1, 2019.

Communicated by Cheng-Hsiung Hsu.

2010 Mathematics Subject Classification. 35K57, 35C07, 92D25.

Key words and phrases. discrete diffusion equations, traveling waves, global stability, weighted energy method.

*Corresponding author. 
$\sum_{i \in \mathbb{Z}} K(i) g(u(t-\tau, x-i))$ involves an infinite summation accounting for the non-local interaction, $K$ is the non-negative weighted function satisfying

$$
K(i)=K(-i) \geq 0, \quad \sum_{i \in \mathbb{Z}} K(i)=1 \quad \text { and } \quad \sum_{i \in \mathbb{Z}} K(i) e^{-\lambda i}<\infty
$$

for any $\lambda>0$, and $g(\cdot)$ is the birth rate function satisfying

(G1) $g(0)=0, g\left(u_{+}\right)=u_{+}$for some positive constant $u_{+}, g(u)>u$ for $u \in\left(0, u_{+}\right)$, and $g^{\prime}(0)>1$ and $g^{\prime}\left(u_{+}\right)<1$;

(G2) $g(u)>0$ has only one positive local maximum at the point $u_{*} \in\left(0, u_{+}\right)$, and $g(u)$ is increasing on $\left[0, u_{*}\right]$ and decreasing on $\left[u_{*},+\infty\right)$;

(G3) $g \in C^{2}[0, \infty)$ and $\left|g^{\prime}(u)\right| \leq g^{\prime}(0)$ for $u \in[0, \infty)$.

By (G1), we see that the equation (1.1) admits two constant equilibria $u=u_{-}=0$ and $u=u_{+}$, where 0 is unstable and $u_{+}$is stable. (G2) shows that $g(u)$ is not monotone for $u \in\left[0, u_{+}\right]$. Throughout this paper, we assume that

$$
\lim _{x \rightarrow \pm \infty} u_{0}(s, x)=u_{ \pm} \quad \text { uniformly in } s \in[-\tau, 0] .
$$

A traveling wave of (1.1) is a special solution of the form $u(t, x)=\phi(x+c t)$ connecting two equilibria $u_{-}$to $u_{+}$, where $c>0$ is the wave speed. The wave profile equation of (1.1) is

$$
c \phi^{\prime}(\xi)-\mathcal{D}_{2}[\phi](\xi)+\phi(\xi)=\sum_{i \in \mathbb{Z}} K(i) g(\phi(\xi-c \tau-i)), \quad \phi(-\infty)=0, \quad \phi(+\infty)=u_{+}
$$

where $\xi=x+c t,{ }^{\prime}=d / d \xi, \mathcal{D}_{2}[\phi](\xi)=d[\phi(\xi+1)-2 \phi(\xi)+\phi(\xi-1)]$. Moreover, if $\phi(\xi)$ is monotone in $\xi \in \mathbb{R}$, then it is called a traveling wavefront.

Equation (1.1) is a continuum version of the following lattice differential equation

$$
\frac{\partial u_{n}(t)}{\partial t}=d\left[u_{n+1}(t)-2 u_{n}(t)+u_{n-1}(t)\right]-u_{n}(t)+\sum_{i \in \mathbb{Z}} K(i) g\left(u_{n-i}(t-\tau)\right) .
$$

Note that equations (1.1) and (1.5) possess the same wave profile equation (1.4). To the best of our knowledge, the existence of traveling waves and other properties, such as monotonicity and uniqueness of traveling waves of 1.5 were well studied. We refer the readers to [15, 16] for bistable case, [1, 7, 14, 25] for monotone monostable case, and 5, 6, 27, 30 for nonmonotone monostable case. However, little has been done for the stability of traveling waves of (1.1) and (1.5), when the function $g$ is not monotone. More recently, Yang and Zhang [28 proved that all noncritical traveling waves of (1.1) (waves with speeds $c>c_{*}, c_{*}$ is minimal speed) are time-exponentially stable, when the initial 
perturbations around the waves are small. The method adopted in 28 is the technical weighted energy method $4,11,13,19,20,26,32$. We would like to mention that, Tian et al. 24 and Yang et al. [29], respectively, applied this method to prove the local stability of traveling waves of 11.1 when $K(0)=1$ and $K(i)=0$ for all $i \neq 0$. An interesting but also challenging question is whether these traveling waves of 1.1 with speed $c \geq c_{*}$ are globally stable.

The global stability of traveling waves for various monostable evolution equations has been extensively investigated, see e.g., [2,3, 9, 15, 17, 18, 23, 31], and references therein. There are main two methods: the squeezing technique [3, 15] for a class of discrete reactiondiffusion equations; and the weighted energy method together with the comparison principle developed by Mei and coauthors [17, 18 for the Nicholson's blowflies equations, see also 9] for discrete reaction-diffusion equations with nonlocal delay effects, 23] for a discrete reaction-diffusion competition system. We should point out that the comparison principle is needed in above two methods. However, when the birth rate function $g(u)$ in (1.1) is non-monotone, the comparison principle does not hold, and hence, the above two methods are not valid. In this paper, we shall apply the nonlinear Halanay's inequality, the anti-weighted technique, Fourier's transform and the boundedness estimate of traveling waves to establish the global stability of traveling waves. These methods were inspired by Mei et al. [4, 11,22]. More precisely, the time-exponential decay of $U(t, \xi)$ at $\xi=+\infty$ of transformed equation can be obtained by using nonlinear Halanay's inequality (see Proposition 3.3). To obtain the decay estimate of $U(t, \xi)$ for $\xi \in\left(-\infty, x_{0}\right]$ is a key step of this paper, where $x_{0}>0$ is large enough. We first take the anti-weighted technique to obtain a new equation (3.7). By some observation, the absolute value of the traveling waves of equation (3.7) can be bounded by the positive solution of a linear delayed discrete diffusion equation with constant coefficients, and Fourier's transform can be applied to get the decay estimate for the solution of this linear delayed equation. Then we obtain the decay estimate of $U(t, \xi)$ for $\xi \in\left(-\infty, x_{0}\right]$. Combining above two decay estimates, we prove the global stability for the traveling waves with large speed.

The rest of this paper is organized as follows. In Section 2, we introduce some preliminaries and state our stability result. Section 3 is devoted to proving our main result on the global stability of traveling waves with large speed.

\section{Preliminaries and the stability theorem}

In this section, we first give the equivalent integral form of the initial value problem of (1.1), then recall the existence of traveling waves of (1.1), and finally state the main result on the stability of traveling waves. 
First, we consider the initial value problem 1.1 with $(1.2)$, i.e., (2.1)

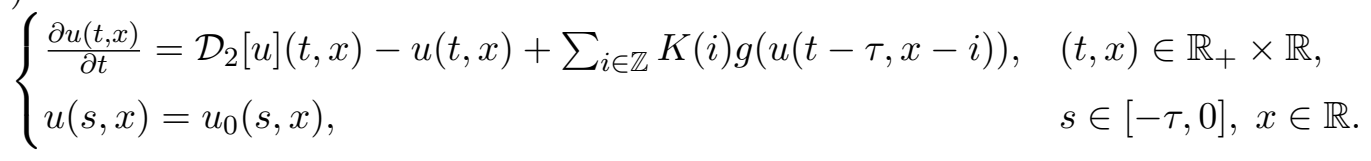

According to [10], with aid of the modified Bessel functions, the solution to the initial value problem

$$
\begin{cases}\frac{\partial u}{\partial t}(t, x)=d[u(t, x+1)-2 u(t, x)+u(t, x-1)], & (t, x) \in \mathbb{R}_{+} \times \mathbb{R}, \\ u(0, x)=u_{0}(x), & x \in \mathbb{R}\end{cases}
$$

can be expressed by

$$
u(t, x)=\left(S(t) u_{0}\right)(x)=e^{-2 d t} \sum_{m=-\infty}^{\infty} \mathbf{I}_{m}(2 d t) u_{0}(x-m)
$$

where $u_{0}(\cdot) \in L^{\infty}(\mathbb{R}), \mathbf{I}_{m}, m \geq 0$ are defined as

$$
\mathbf{I}_{m}(t)=\sum_{k=0}^{\infty} \frac{(t / 2)^{m+2 k}}{k !(m+k) !}
$$

and $\mathbf{I}_{m}(t)=\mathbf{I}_{-m}(t)$ for $m<0$. Moreover,

$$
\mathbf{I}_{m}^{\prime}(t)=\frac{1}{2}\left[\mathbf{I}_{m+1}(t)+\mathbf{I}_{m-1}(t)\right], \quad \forall t \geq 0, m \in \mathbb{Z},
$$

and $\mathbf{I}_{m}(0)=0$ for $m \neq 0$ while $\mathbf{I}_{0}(0)=1$, and $\mathbf{I}_{m}(t) \geq 0$ for any integer $m$ and $t \geq 0$. It is easy to see that

$$
e^{-t} \sum_{m=-\infty}^{\infty} \mathbf{I}_{m}(t)=e^{-t}\left[\mathbf{I}_{0}(t)+2 \mathbf{I}_{1}(t)+2 \mathbf{I}_{3}(t)+\cdots\right]=1
$$

Clearly, (2.1) is equivalent to

$$
\begin{aligned}
& u(t, x) \\
= & e^{-(2 d+1) t} \sum_{m=-\infty}^{\infty} \mathbf{I}_{m}(2 d t) u_{0}(0, x-m) \\
& +\sum_{m=-\infty}^{\infty} \int_{0}^{t} e^{-(2 d+1)(t-s)} \mathbf{I}_{m}(2 d(t-s))\left(\sum_{i \in \mathbb{Z}} K(i) g(u(s-\tau, x-m-i))\right) d s .
\end{aligned}
$$

In fact, by $(\mathrm{G} 2)$, one has $g(u) \leq g\left(u_{*}\right)$ for $u \in[0,+\infty)$. Then by [10, Lemma 2.1], we can 
differentiate the series on $t$ variable in (2.4). Then we obtain

$$
\begin{aligned}
& \frac{\partial u(t, x)}{\partial t} \\
= & -(2 d+1) e^{-(2 d+1) t} \sum_{m=-\infty}^{\infty} \mathbf{I}_{m}(2 d t) u_{0}(0, x-m) \\
& +e^{-(2 d+1) t} \sum_{m=-\infty}^{\infty} 2 d \mathbf{I}_{m}^{\prime}(2 d t) u_{0}(0, x-m) \\
& +\sum_{m=-\infty}^{\infty} I_{m}(0) \sum_{i \in \mathbb{Z}} K(i) g(u(t-\tau, x-m-i)) \\
& -(2 d+1) \sum_{m=-\infty}^{\infty} \int_{0}^{t} e^{-(2 d+1)(t-s)} \mathbf{I}_{m}(2 d(t-s))\left(\sum_{i \in \mathbb{Z}} K(i) g(u(s-\tau, x-m-i))\right) d s \\
& +\sum_{m=-\infty}^{\infty} \int_{0}^{t} e^{-(2 d+1)(t-s)} 2 d \mathbf{I}_{m}^{\prime}(2 d(t-s))\left(\sum_{i \in \mathbb{Z}} K(i) g(u(s-\tau, x-m-i))\right) d s \\
= & d[u(t, x+1)-2 u(t, x)+u(t, x-1)]-u(t, x)+\sum_{i \in \mathbb{Z}} K(i) g(u(t-\tau, x-i)),
\end{aligned}
$$

where we have used the recurrence relation $(2.2)$.

The characteristic function for (1.4) with respect to the trivial equilibrium 0 can be represented by

$$
\mathcal{P}(c, \lambda)=c \lambda-d\left(e^{\lambda}+e^{-\lambda}-2\right)+1-g^{\prime}(0) G(\lambda)
$$

where

$$
G(\lambda)=\sum_{i \in \mathbb{Z}} K(i) e^{-\lambda(i+c \tau)}<\infty .
$$

One can easily show that the following result holds.

Lemma 2.1. Assume that $g^{\prime}(0)>1$. Then there exist $\lambda_{*}>0$ and $c_{*}>0$ such that

$$
\mathcal{P}\left(c_{*}, \lambda_{*}\right)=0 \quad \text { and }\left.\quad \frac{\partial}{\partial \lambda} \mathcal{P}\left(c_{*}, \lambda\right)\right|_{\lambda=\lambda_{*}}=0
$$

Furthermore, if $c>c_{*}$, then $\mathcal{P}(c, \lambda)=0$ has two distinct positive real roots $\lambda_{1}(c)$ and $\lambda_{2}(c)$ with $\lambda_{1}(c)<\lambda_{*}<\lambda_{2}(c)$, and $\mathcal{P}(c, \lambda)>0$ for $\lambda \in\left(\lambda_{1}(c), \lambda_{2}(c)\right)$.

The existence of traveling waves has been obtained by Yang and Zhang, see 28, Theorem 2.3].

Proposition 2.2. Assume that (G1)-(G3) hold. Then for every $c>c_{*}$, (1.1) admits a traveling wave $u(t, x)=\phi(x+c t)$ satisfying $\phi(-\infty)=0$ and $u_{-}^{*}<\liminf _{\xi \rightarrow+\infty} \phi(\xi) \leq$ $\lim \sup _{\xi \rightarrow+\infty} \phi(\xi) \leq u_{+}^{*}$ for some positive constant $u_{+}^{*}>u_{+}$. 
Remark 2.3. We should point out if we further assume that the following assumption (G4) holds, then $\phi(+\infty)=u_{+}$.

(G4) $g(u)<2 u_{+}-u$ for $u \in\left[u_{-}^{*}, u_{+}\right)$and $g(u)>2 u_{+}-u$ for $u \in\left(u_{+}, u_{+}^{*}\right]$, where $0<u_{-}^{*} \leq$ $u_{+} \leq u_{+}^{*}$ and $g_{+}\left(u_{+}^{*}\right)=d u_{+}^{*}$ and $g_{-}\left(u_{-}^{*}\right)=d u_{-}^{*}$, where $g_{+}(u):=\min _{w \in[0, u]} g(w)$ and $g_{-}(u):=\min _{w \in\left[u, u_{+}^{*}\right]} g(w)$.

Notations. $C>0$ denotes a generic constant, while $C_{i}(i=1,2, \ldots)$ represents a specific constant. Let $I$ be an interval, typically $I=\mathbb{R}$. Denote by $L^{1}(I)$ the space of integrable functions defined on $I$, and $W^{k, 1}(I)(k \geq 0)$ the Sobolev space of the $L^{1}$-functions $f(x)$ defined on the interval $I$ whose derivatives $\frac{d^{n}}{d x^{n}} f(n=1, \ldots, k)$ also belong to $L^{1}(I)$. Let $L_{w}^{1}(I)$ be the weighted $L^{1}$-space with a weight function $w(x)>0$ and its norm is defined by

$$
\|f\|_{L_{w}^{1}(I)}=\int_{I} w(x)|f(x)| d x,
$$

$W_{w}^{k, 1}(I)$ be the weighted Sobolev space with the norm given by

$$
\|f\|_{W_{w}^{k, 1}(I)}=\sum_{i=0}^{k} \int_{I} w(x)\left|\frac{d^{i} f(x)}{d x^{i}}\right| d x .
$$

Let $T>0$ be a number and $\mathcal{B}$ be a Banach space. We denote by $C([0, T] ; \mathcal{B})$ the space of the $\mathcal{B}$-valued continuous functions on $[0, T]$, and by $L^{1}([0, T] ; \mathcal{B})$ the space of the $\mathcal{B}$-valued $L^{1}$-functions on $[0, T]$. The corresponding spaces of the $\mathcal{B}$-valued functions on $[0, \infty)$ are defined similarly. For any function $f(x)$, its Fourier transform is defined by

$$
\mathcal{F}[f](\eta)=\widehat{f}(\eta)=\int_{\mathbb{R}} e^{-\mathbf{i} x \eta} f(x) d x
$$

and the inverse Fourier transform is given by

$$
\mathcal{F}^{-1}[\widehat{f}](x)=\frac{1}{2 \pi} \int_{\mathbb{R}} e^{\mathbf{i} x \eta} \widehat{f}(\eta) d \eta,
$$

where $\mathbf{i}$ is the imaginary unit, $\mathbf{i}^{2}=-1$.

Define a weight function

$$
\omega(\xi):=e^{-2 \lambda \xi}, \quad \xi \in \mathbb{R},
$$

where $\lambda=c_{*}$. Notice that $\lim _{\xi \rightarrow-\infty} \omega(\xi)=+\infty$ and $\lim _{\xi \rightarrow+\infty} \omega(\xi)=0$, since $\lambda=c_{*}>0$.

For the sake of simplicity, we denote

$$
L_{1}:=d\left(e^{c_{*}}+e^{-c_{*}}-2\right), \quad L_{2}:=e^{d\left(e^{c_{*}}+e^{-c_{*}}\right) \tau} g^{\prime}(0) \sum_{i \in \mathbb{Z}} K(i) e^{-c_{*} i} .
$$

Now we state the stability result of traveling waves of 1.1 with a general nonmonotone function $g(u)$. 
Theorem 2.4 (Global stability). Assume that (G1)-(G3) hold. For any given traveling wave $\phi(x+c t)=\phi(\xi)$ connecting 0 and $u_{+}$with

$$
c>\widetilde{c}:=\max \left\{c_{*}, \frac{L_{1}-1+L_{2}}{c_{*}}\right\},
$$

whether it is monotone or non-monotone, when the initial perturbation satisfies $u_{0}-\phi \in$ $\mathcal{C}_{\text {unif }}[-\tau, 0] \cap C\left([-\tau, 0] ; W_{\omega}^{1,1}(\mathbb{R})\right)$ and $\partial_{s}\left(u_{0}-\phi\right) \in L^{1}\left([-\tau, 0] ; L_{\omega}^{1}(\mathbb{R})\right)$, we have the following global stability

$$
\sup _{x \in \mathbb{R}}|u(t, x)-\phi(x+c t)| \leq C e^{-\mu t},
$$

where $\mu$ and $C$ are positive numbers, and $\mathcal{C}_{\text {unif }}[-\tau, T]$ is the uniformly continuous space, for $0<T \leq \infty$, defined by

$$
\begin{aligned}
& \mathcal{C}_{\text {unif }}[-\tau, T] \\
= & \left\{u \in C([-\tau, T] \times \mathbb{R}) \text { such that } \lim _{x \rightarrow+\infty} u(t, x) \text { exists uniformly in } t \in[-\tau, T]\right\} .
\end{aligned}
$$

\section{The global stability of traveling waves}

In this section, we are devoted to the proof of global stability of those monotone or nonmonotone traveling waves of 1.1 with speed $c>\widetilde{c}$, when $g$ is non-monotone.

Let $\phi(x+c t)=\phi(\xi)$ be any given traveling wave with speed $c>c_{*}$, and define

$$
\begin{aligned}
U(t, \xi) & :=u(t, x)-\phi(x+c t)=u(t, \xi-c t)-\phi(\xi), \\
U_{0}(s, \xi) & :=u_{0}(s, x)-\phi(x+c s) .
\end{aligned}
$$

Then it follows from (1.1) and (1.4) that $U(t, \xi)$ satisfies

$$
\begin{cases}\frac{\partial U}{\partial t}+c \frac{\partial U}{\partial \xi}-\mathcal{D}_{2}[U]+U=\sum_{i \in \mathbb{Z}} K(i) Q(U(t-\tau, \xi-c \tau-i)), & (t, \xi) \in \mathbb{R}_{+} \times \mathbb{R} \\ U(s, \xi)=U_{0}(s, \xi), & s \in[-\tau, 0], \xi \in \mathbb{R}\end{cases}
$$

where

$$
Q(U):=g(\phi+U)-g(\phi)=g^{\prime}(\widetilde{\phi}) U
$$

for some $\widetilde{\phi}$ between $\phi$ and $\phi+U$, with $\phi=\phi(\xi-c \tau-i)$ and $U=U(t-\tau, \xi-c \tau-i)$ for $c>c_{*}$.

We first prove the existence and uniqueness of solution to the initial value problem (3.1) in the uniformly continuous space $\mathcal{C}_{\text {unif }}[-\tau, \infty)$.

Lemma 3.1. Assume that (G1)-(G3) hold. If the initial perturbation $U_{0} \in \mathcal{C}_{\text {unif }}[-\tau, 0]$ for $c>c_{*}$, then the solution $U(t, \xi)$ of perturbed equation (3.1) is unique and time-globally exists in $\mathcal{C}_{\text {unif }}[-\tau, \infty)$. 
Proof. Let $V(t, x)=u(t, x)-\phi(x+c t)$. It is clear that $V(t, x)=U(t, \xi)$ and satisfies

$$
\begin{cases}\frac{\partial V}{\partial t}-\mathcal{D}_{2}[V]+V=\sum_{i \in \mathbb{Z}} K(i) Q(V(t-\tau, x-i)), & (t, x) \in \mathbb{R}_{+} \times \mathbb{R}, \\ V(s, x)=u_{0}(s, x)-\phi(x+c s):=V_{0}(s, x), & (s, x) \in[-\tau, 0] \times \mathbb{R}\end{cases}
$$

Thus, the global existence and uniqueness of solutions of (3.1) are transformed into that of 3.3 .

When $t \in[0, \tau]$, we have $t-\tau \in[-\tau, 0]$ and $V(t-\tau, x-i)=V_{0}(t-\tau, x-i)$. Consequently, the solution of $(3.3)$ can be explicitly and uniquely solved by

$$
\begin{aligned}
& V(t, x) \\
= & e^{-(2 d+1) t} \sum_{m=-\infty}^{\infty} \mathbf{I}_{m}(2 d t) V_{0}(0, x-m) \\
& +\sum_{m=-\infty}^{\infty} \int_{0}^{t} e^{-(2 d+1)(t-s)} \mathbf{I}_{m}(2 d(t-s))\left(\sum_{i \in \mathbb{Z}} K(i) Q\left(V_{0}(s-\tau, x-m-i)\right)\right) d s
\end{aligned}
$$

for $t \in[0, \tau]$.

Since $U_{0} \in \mathcal{C}_{\text {unif }}[-\tau, 0], \lim _{\xi \rightarrow+\infty} U_{0}(\xi, t)$ exists uniformly in $t \in[-\tau, 0]$, which implies $\lim _{x \rightarrow+\infty} V_{0}(t, x)$ exists uniformly in $t \in[-\tau, 0]$. Denote $V_{0}(t, \infty)=\lim _{x \rightarrow+\infty} V_{0}(t, x)$. Taking the limit $x \rightarrow+\infty$ to 3.4 yields

$$
\begin{aligned}
& \lim _{x \rightarrow+\infty} V(t, x) \\
= & e^{-(2 d+1) t} \sum_{m=-\infty}^{\infty} \mathbf{I}_{m}(2 d t) \lim _{x \rightarrow+\infty} V_{0}(0, x-m) \\
& +\sum_{m=-\infty}^{\infty} \int_{0}^{t} e^{-(2 d+1)(t-s)} \mathbf{I}_{m}(2 d(t-s))\left(\sum_{i \in \mathbb{Z}} K(i) \lim _{x \rightarrow+\infty} Q\left(V_{0}(s-\tau, x-m-i)\right)\right) d s \\
= & e^{-t} V_{0}(0, \infty)+\int_{0}^{t} e^{-(t-s)} Q\left(V_{0}(s-\tau, \infty)\right) \sum_{m=-\infty}^{\infty} e^{-2 d(t-s)} \mathbf{I}_{m}(2 d(t-s)) d s \\
= & : \mathcal{V}_{1}(t) \quad \text { uniformly in } t \in[0, \tau] .
\end{aligned}
$$

Here we use the fact that $\sum_{i \in \mathbb{Z}} K(i)=1$ and $(2.3)$. Thus, we obtain that $V \in \mathcal{C}_{\text {unif }}[-\tau, \tau]$, i.e., $U \in \mathcal{C}_{\text {unif }}[-\tau, \tau]$.

When $t \in[\tau, 2 \tau]$, equation 3.3 with the initial data $V(s, x)$ for $s \in[0, \tau]$ is still linear because the source term $\sum_{i \in \mathbb{Z}} K(i) Q(V(t-\tau, x-i))$ is known due to $t-\tau \in[0, \tau]$ and $V(t-\tau, x)$ is solved in (3.4). Hence, the solution $V(t, x)$ for $t \in[\tau, 2 \tau]$ is uniquely and 
explicitly given by

$$
\begin{aligned}
V(t, x)= & e^{-(2 d+1)(t-\tau)} \sum_{m=-\infty}^{\infty} \mathbf{I}_{m}(2 d(t-\tau)) V(\tau, x-m) \\
& +\sum_{m=-\infty}^{\infty} \int_{\tau}^{t} e^{-(2 d+1)(t-s)} \mathbf{I}_{m}(2 d(t-s))\left(\sum_{i \in \mathbb{Z}} K(i) Q(V(s-\tau, x-m-i))\right) d s .
\end{aligned}
$$

Similarly, by (3.5), we have

$$
\begin{aligned}
& \lim _{x \rightarrow+\infty} V(t, x) \\
= & e^{-(2 d+1)(t-\tau)} \sum_{m=-\infty}^{\infty} \mathbf{I}_{m}(2 d(t-\tau)) \lim _{x \rightarrow+\infty} V(\tau, x-m) \\
& +\sum_{m=-\infty}^{\infty} \int_{\tau}^{t} e^{-(2 d+1)(t-s)} \mathbf{I}_{m}(2 d(t-s))\left(\sum_{i \in \mathbb{Z}} K(i) \lim _{x \rightarrow+\infty} Q(V(s-\tau, x-m-i))\right) d s \\
= & e^{-(t-\tau)} \mathcal{V}_{1}(\tau)+\int_{\tau}^{t} e^{-(t-s)} Q\left(\mathcal{V}_{1}(s-\tau)\right) \sum_{m=-\infty}^{\infty} e^{-2 d(t-s)} \mathbf{I}_{m}(2 d(t-s)) d s \\
= & : \mathcal{V}_{2}(t) \quad \text { uniformly in } t \in[\tau, 2 \tau] .
\end{aligned}
$$

Repeating the above procedure for $t \in[n \tau,(n+1) \tau]$ with $n=2,3, \ldots$, we can obtain that $U \in \mathcal{C}_{\text {unif }}[-\tau,(n+1) \tau]$ uniquely exists. Step by step, we can finally prove the global existence and uniqueness of solution $U \in \mathcal{C}_{\text {unif }}[-\tau,+\infty)$.

Now we state the stability result for the perturbed equation (3.1), which automatically implies Theorem 2.4 .

Proposition 3.2. Assume that (G1)-(G3) hold. If the initial perturbation

$$
U_{0} \in \mathcal{C}_{\text {unif }}[-\tau, 0] \cap C\left([-\tau, 0] ; W_{\omega}^{1,1}(\mathbb{R})\right), \quad \partial_{s} U_{0} \in L^{1}\left([-\tau, 0] ; L_{\omega}^{1}(\mathbb{R})\right),
$$

then when $c>\widetilde{c}$, it holds

$$
\sup _{\xi \in \mathbb{R}}|U(t, \xi)| \leq C e^{-\mu t}, \quad t>0
$$

for some positive constant $\mu$.

Proposition 3.2 is a straightforward consequence of the following Propositions 3.3 and 3.11. In the rest of this section, we always assume the assumptions (G1)-(G3) hold.

Proposition 3.3. Assume that $U_{0} \in \mathcal{C}_{\text {unif }}[-\tau, 0]$. Then there exists a large number $x_{0} \gg 1$ such that the solution $U(t, \xi)$ of 3.1 satisfies

$$
\sup _{\xi \in\left[x_{0}, \infty\right)}|U(t, \xi)| \leq C e^{-\mu_{1} t}, \quad t>0
$$

for some $0<\mu_{1}<1$. 
Proof. Since $U_{0} \in \mathcal{C}_{\text {unif }}[-\tau, 0]$, by Lemma 3.1. we have $U \in \mathcal{C}_{\text {unif }}[-\tau, \infty)$, which implies $\lim _{\xi \rightarrow+\infty} U(t, \xi)=U(t, \infty)=: z(t)$ exists uniformly for $t \in[-\tau, \infty)$. Taking the limit $\xi \rightarrow \infty$ to 3.1 , one has

$$
\frac{d}{d t} z(t)+z(t)-g^{\prime}\left(u_{+}\right) z(t-\tau)=P(z(t-\tau)), \quad z(s)=z_{0}(s), \quad s \in[-\tau, 0],
$$

where

$$
P(z)=g\left(u_{+}+z\right)-g\left(u_{+}\right)-g^{\prime}\left(u_{+}\right) z .
$$

It follows from the Taylor expansion that $|P(z)| \leq C|z|^{2}$ for some positive constant $C$. By the nonlinear Halanay's inequality given in [13], we obtain

$$
|U(t, \infty)|=|z(t)| \leq C e^{-\mu_{1} t}, \quad t>0
$$

for some constant $0<\mu_{1}<1$, provided $\left\|z_{0}\right\|_{L^{\infty}} \ll 1$.

By the continuity and the uniform convergence of $U(t, \xi)$ as $\xi \rightarrow+\infty$, there exists a large number $x_{0} \gg 1$ such that (3.6) implies

$$
\sup _{\xi \in\left[x_{0}, \infty\right)}|U(t, \xi)| \leq C e^{-\mu_{1} t}, \quad t>0,
$$

provided $\sup _{\xi \in\left[x_{0},+\infty\right)}\left|U_{0}(s, \xi)\right| \ll 1$ for $s \in[-\tau, 0]$. Such a smallness for the initial perturbation $U_{0}$ near $\xi \rightarrow+\infty$ can be automatically verified, since $\lim _{x \rightarrow+\infty} u_{0}(s, x)=u_{+}$ (see $(1.3)$ ), which implies $\lim _{\xi \rightarrow+\infty} U_{0}(s, \xi)=\lim _{\xi \rightarrow+\infty}\left[u_{0}(s, \xi)-\phi(\xi)\right]=u_{+}-u_{+}=0$ uniformly for $s \in[-\tau, 0]$.

It remains to establish a priori decay estimate of $\sup _{\xi \in\left(-\infty, x_{0}\right]}|U(t, \xi)|$. We shall use the anti-weighted technique [4,11] together with Fourier's transform to treat this problem. Define

$$
\widetilde{U}(t, \xi)=\sqrt{\omega(\xi)} U\left(t, \xi+x_{0}\right)=e^{-\lambda \xi} U\left(t, \xi+x_{0}\right),
$$

where $\lambda=c_{*}$. It is easy to see that $\widetilde{U}(t, \xi)$ satisfies

$$
\begin{cases}\frac{\partial \widetilde{U}}{\partial t}+c \frac{\partial \widetilde{U}}{\partial \xi}+c_{1} \widetilde{U}(t, \xi)-d e^{\lambda} \widetilde{U}(t, \xi+1)-d e^{-\lambda} \widetilde{U}(t, \xi-1) & \\ =\sum_{i \in \mathbb{Z}} K(i) \widetilde{Q}(\widetilde{U}(t-\tau, \xi-c \tau-i)), & (t, \xi) \in \mathbb{R}_{+} \times \mathbb{R} \\ \widetilde{U}(s, \xi)=\sqrt{\omega(\xi)} U\left(s, \xi+x_{0}\right)=: \widetilde{U}_{0}(s, \xi), & s \in[-\tau, 0], \xi \in \mathbb{R}\end{cases}
$$

where $c_{1}=c \lambda+2 d+1$ and

$$
\widetilde{Q}(\widetilde{U})=e^{-\lambda \xi} Q(U) .
$$

By (3.2), we obtain

$$
\begin{aligned}
\widetilde{Q}(\widetilde{U}(t-\tau, \xi-c \tau-i)) & =e^{-\lambda \xi} Q\left(U\left(t-\tau, \xi+x_{0}-c \tau-i\right)\right) \\
& =e^{-\lambda \xi} g^{\prime}(\widetilde{\phi}) U\left(t-\tau, \xi+x_{0}-c \tau-i\right) \\
& =e^{-\lambda(i+c \tau)} g^{\prime}(\widetilde{\phi}) \widetilde{U}(t-\tau, \xi-c \tau-i)
\end{aligned}
$$


for some function $\widetilde{\phi}$ between $\phi$ and $\phi+U$. By (G3), we further have

$$
|\widetilde{Q}(\widetilde{U}(t-\tau, \xi-c \tau-i))| \leq g^{\prime}(0) e^{-\lambda(i+c \tau)}|\widetilde{U}(t-\tau, \xi-c \tau-i)|
$$

Since $g^{\prime}(s)$ can be negative for $s \in\left(0, u_{+}\right)$, then the solution $\widetilde{U}$ of 3.7$)$ with the nonlinear term (3.8) may be oscillation around $u_{+}$when the time delay $\tau$ is large. At the same time, the comparison principle may not hold for (3.7). Hence, the monotonic technique cannot be applied. Note that the coefficient $g^{\prime}(\widetilde{\phi})$ in $(3.8)$ is variable. As such, we are unable to derive the decay estimate directly by applying Fourier's transform. In this paper, we shall provide some new idea to establish the decay estimate of $\sup _{\xi \in\left(-\infty, x_{0}\right]}|U(\xi, t)|$. By replacing $g^{\prime}(\widetilde{\phi})$ in $(3.7)$ with $g^{\prime}(0)$, we obtain a linear delayed discrete diffusion equation

$$
\begin{cases}\frac{\partial U^{+}}{\partial t}+c \frac{\partial U^{+}}{\partial \xi}+c_{1} U^{+}(t, \xi)-d e^{\lambda} U^{+}(t, \xi+1)-d e^{-\lambda} U^{+}(t, \xi-1) & \\ =g^{\prime}(0) \sum_{i \in \mathbb{Z}} K(i) e^{-\lambda(i+c \tau)} U^{+}(t-\tau, \xi-c \tau-i), & (t, \xi) \in \mathbb{R}_{+} \times \mathbb{R}, \\ U^{+}(s, \xi)=U_{0}^{+}(s, \xi) \geq 0, & s \in[-\tau, 0], \xi \in \mathbb{R} .\end{cases}
$$

We first prove that the solution $\widetilde{U}$ of 3.7 can be bounded by the solution $U^{+}$of 3.9 , i.e., $|\widetilde{U}(t, \xi)| \leq U^{+}(t, \xi)$ for $(t, \xi) \in \mathbb{R}_{+} \times \mathbb{R}$. Since $(3.9)$ is a linear equation, we can apply Fourier's transform to investigate the decay estimate of $U^{+}$. Then the decay estimate of $\widetilde{U}$ can be obtained.

In order to obtain the crucial boundedness estimate of $\widetilde{U}$, we need the following maximum principle.

Lemma 3.4. Let $T>0$. For any $a_{1}, a_{2} \in \mathbb{R}$ and $\nu>0$, if the bounded function $v$ satisfies

$$
\begin{cases}\frac{\partial v}{\partial t}+a_{1} \frac{\partial v}{\partial \xi}+a_{2} v-d e^{\nu} v(t, \xi+1)-d e^{-\nu} v(t, \xi-1) \geq 0, & (t, \xi) \in(0, T] \times \mathbb{R}, \\ v(0, \xi) \geq 0, & \xi \in \mathbb{R},\end{cases}
$$

then $v(t, \xi) \geq 0$ for all $(t, \xi) \in(0, T] \times \mathbb{R}$.

Proof. Let $\widetilde{v}(t, \xi)=v\left(t, \xi+a_{1} t\right)$. Then by 3.10$), \widetilde{v}(\xi, t)$ satisfies

$$
\begin{cases}\frac{\partial \widetilde{v}}{\partial t}+a_{2} \widetilde{v}-d e^{\nu} \widetilde{v}(t, \xi+1)-d e^{-\nu} \widetilde{v}(t, \xi-1) \geq 0, & (t, \xi) \in(0, T] \times \mathbb{R} \\ \widetilde{v}(0, \xi)=v(0, \xi) \geq 0, & \xi \in \mathbb{R} .\end{cases}
$$

Let $w(t, \xi)=e^{k t} \widetilde{v}(t, \xi)$, where $k>0$ is chosen such that $p_{0}:=k-a_{2}>0$. Then we have

$$
\frac{\partial w(t, \xi)}{\partial t} \geq d e^{\nu} \widetilde{w}(t, \xi+1)+d e^{-\nu} \widetilde{w}(t, \xi-1)+p_{0} w(t, \xi), \quad(t, \xi) \in(0, T] \times \mathbb{R} .
$$

Denote $0<T_{0}<\min \left\{T, \frac{1}{2\left(d e^{\nu}+d e^{-\nu}+p_{0}\right)}\right\}$. We now prove that $w \geq 0$ in $\left(0, T_{0}\right) \times \mathbb{R}$. Suppose on the contrary that there are $\widetilde{t} \in\left(0, T_{0}\right)$ and $\widetilde{\xi} \in \mathbb{R}$ such that $w(\widetilde{t}, \widetilde{\xi})<$ 
0 . Then by the assumption of the lemma, there exists $t^{*} \in\left(0, T_{0}\right)$ such that $w_{\text {inf }}:=$ $\inf _{(t, \xi) \in\left[0, t^{*}\right] \times \mathbb{R}} w(t, \xi)<0$. Observe that there exists $\left(t_{n}, \xi_{n}\right) \in\left(0, t^{*}\right] \times \mathbb{R}$ such that $w\left(t_{n}, \xi_{n}\right) \rightarrow w_{\text {inf }}$ as $n \rightarrow \infty$. Integrating (3.11) from 0 to $t_{n}$, we obtain

$$
\begin{aligned}
w\left(t_{n}, \xi_{n}\right)-w\left(0, \xi_{n}\right) \geq & d \int_{0}^{t_{n}}\left(e^{\nu} w\left(s, \xi_{n}+1\right)+e^{-\nu} w\left(s, \xi_{n}-1\right)\right) d s \\
& +\int_{0}^{t_{n}} p_{0} w\left(s, \xi_{n}\right) d s \\
\geq & \left(d e^{\nu}+d e^{-\nu}+p_{0}\right) w_{\text {inf }} t_{n} \\
\geq & \left(d e^{\nu}+d e^{-\nu}+p_{0}\right) w_{\text {inf }} t^{*} .
\end{aligned}
$$

Note that $w\left(0, \xi_{n}\right) \geq 0$ for $n=1,2, \ldots$ Then we have $w\left(t_{n}, \xi_{n}\right) \geq\left(d e^{\nu}+d e^{-\nu}+p_{0}\right) w_{\text {inf }} t^{*}$. Letting $n \rightarrow \infty$, we obtain

$$
w_{\mathrm{inf}} \geq\left(d e^{\nu}+d e^{-\nu}+p_{0}\right) w_{\mathrm{inf}} t^{*}>\frac{1}{2} w_{\mathrm{inf}},
$$

a contradiction. Hence, $v(t, \xi) \geq 0$ for all $(t, \xi) \in(0, T] \times \mathbb{R}$.

Lemma 3.5. If $U_{0}^{+}(s, \xi) \geq 0$ for $(s, \xi) \in[-\tau, 0] \times \mathbb{R}$, then $U^{+}(t, \xi) \geq 0$ for $(t, \xi) \in$ $[-\tau,+\infty) \times \mathbb{R}$.

Proof. For $t \in[0, \tau]$, we have $t-\tau \in[-\tau, 0]$ and

$$
\begin{aligned}
& g^{\prime}(0) \sum_{i \in \mathbb{Z}} K(i) e^{-\lambda(i+c \tau)} U^{+}(t-\tau, \xi-c \tau-i) \\
= & g^{\prime}(0) \sum_{i \in \mathbb{Z}} K(i) e^{-\lambda(i+c \tau)} U_{0}^{+}(t-\tau, \xi-c \tau-i) \geq 0 .
\end{aligned}
$$

Then it follows from 3.9 that

$$
\frac{\partial U^{+}}{\partial t}+c \frac{\partial U^{+}}{\partial \xi}+c_{1} U^{+}(t, \xi)-d e^{\lambda} U^{+}(t, \xi+1)-d e^{-\lambda} U^{+}(t, \xi-1) \geq 0,
$$

which implies $U^{+}(t, \xi) \geq 0$ for $t \in[0, \tau]$ due to Lemma 3.4. Repeating this procedure step by step, we can prove that $U^{+}(t, \xi) \geq 0$ for $t \in[n \tau,(n+1) \tau]$. Furthermore, we have $U^{+}(t, \xi) \geq 0$ for $(t, \xi) \in \mathbb{R}_{+} \times \mathbb{R}$.

The following result shows the boundedness estimate for the solution $\widetilde{U}(t, \xi)$ of (3.7).

Lemma 3.6. Let $\widetilde{U}(t, \xi)$ and $U^{+}(t, \xi)$ be the solutions of 3.7) and 3.9), respectively. When

$$
\left|\widetilde{U}_{0}(s, \xi)\right| \leq U_{0}^{+}(s, \xi), \quad(s, \xi) \in[-\tau, 0] \times \mathbb{R},
$$

then

$$
|\widetilde{U}(t, \xi)| \leq U^{+}(t, \xi), \quad(t, \xi) \in \mathbb{R}_{+} \times \mathbb{R}
$$


Proof. We first prove $|\widetilde{U}(t, \xi)| \leq U^{+}(t, \xi)$ for $(t, \xi) \in[0, \tau] \times \mathbb{R}$. When $t \in[0, \tau]$, namely, $t-\tau \in[-\tau, 0]$, by 3.12 , we have

$$
\begin{aligned}
|\widetilde{U}(t-\tau, \xi-c \tau-i)| & =\left|\widetilde{U}_{0}(t-\tau, \xi-c \tau-i)\right| \\
& \leq U_{0}^{+}(t-\tau, \xi-c \tau-i)=U^{+}(t-\tau, \xi-c \tau-i) .
\end{aligned}
$$

Let

$$
V^{-}(t, \xi):=U^{+}(t, \xi)-\widetilde{U}(t, \xi) \quad \text { and } \quad V^{+}(t, \xi):=U^{+}(t, \xi)+\widetilde{U}(t, \xi) .
$$

By (3.7), (3.8) and (3.9), we see that $V^{-}(t, \xi)$ satisfies

$$
\begin{aligned}
& \frac{\partial V^{-}}{\partial t}+c \frac{\partial V^{-}}{\partial \xi}+c_{1} V^{-}(t, \xi)-d e^{\lambda} V^{-}(t, \xi+1)-d e^{-\lambda} V^{-}(t, \xi-1) \\
= & g^{\prime}(0) \sum_{i \in \mathbb{Z}} K(i) e^{-\lambda(i+c \tau)} U^{+}(t-\tau, \xi-c \tau-i) \\
& -g^{\prime}(\widetilde{\phi}) \sum_{i \in \mathbb{Z}} K(i) e^{-\lambda(i+c \tau)} \widetilde{U}(t-\tau, \xi-c \tau-i) \\
\geq & g^{\prime}(0) \sum_{i \in \mathbb{Z}} K(i) e^{-\lambda(i+c \tau)} U^{+}(t-\tau, \xi-c \tau-i) \\
& -\sum_{i \in \mathbb{Z}} K(i) e^{-\lambda(i+c \tau)}\left|g^{\prime}(\widetilde{\phi})\right||\widetilde{U}(t-\tau, \xi-c \tau-i)| .
\end{aligned}
$$

Furthermore, by (3.13) and (G3), we obtain from (3.14) that

$$
\frac{\partial V^{-}}{\partial t}+c \frac{\partial V^{-}}{\partial \xi}+c_{1} V^{-}(t, \xi)-d e^{\lambda} V^{-}(t, \xi+1)-d e^{-\lambda} V^{-}(t, \xi-1) \geq 0
$$

for $(t, \xi) \in[0, \tau] \times \mathbb{R}$. Thus, 3.15 with the initial data $V_{0}^{-}(s, \xi)=U_{0}^{+}(s, \xi)-\widetilde{U}_{0}(s, \xi) \geq 0$ reduces to

$$
\frac{\partial V^{-}}{\partial t}+c \frac{\partial V^{-}}{\partial \xi}+c_{1} V^{-}(t, \xi)-d e^{\lambda} V^{-}(t, \xi+1)-d e^{-\lambda} V^{-}(t, \xi-1) \geq 0, \quad V_{0}^{-}(0, \xi) \geq 0 .
$$

By Lemma 3.4, we have

$$
V^{-}(t, \xi)=U^{+}(t, \xi)-\widetilde{U}(t, \xi) \geq 0 \quad \text { for }(t, \xi) \in[0, \tau] \times \mathbb{R} .
$$

On the other hand, $V^{+}(t, \xi)$ satisfies

$$
\begin{aligned}
& \frac{\partial V^{+}}{\partial t}+c \frac{\partial V^{+}}{\partial \xi}+c_{1} V^{+}(t, \xi)-d e^{\lambda} V^{+}(t, \xi+1)-d e^{-\lambda} V^{+}(t, \xi-1) \\
= & g^{\prime}(0) \sum_{i \in \mathbb{Z}} K(i) e^{-\lambda(i+c \tau)} U^{+}(t-\tau, \xi-c \tau-i) \\
& -g^{\prime}(\widetilde{\phi}) \sum_{i \in \mathbb{Z}} K(i) e^{-\lambda(i+c \tau)} \widetilde{U}(t-\tau, \xi-c \tau-i)
\end{aligned}
$$




$$
\begin{aligned}
& \geq g^{\prime}(0) \sum_{i \in \mathbb{Z}} K(i) e^{-\lambda(i+c \tau)} U^{+}(t-\tau, \xi-c \tau-i) \\
& \quad-\sum_{i \in \mathbb{Z}} K(i) e^{-\lambda(i+c \tau)}\left|g^{\prime}(\widetilde{\phi})\right||\widetilde{U}(t-\tau, \xi-c \tau-i)| \\
& \geq 0, \quad t \in[0, \tau] .
\end{aligned}
$$

Similarly, we can prove that 3.17 with the initial data $V_{0}^{+}(s, \xi)=U_{0}^{+}(s, \xi)+\widetilde{U}_{0}(s, \xi) \geq 0$ implies

$$
V^{+}(t, \xi)=U^{+}(t, \xi)+\widetilde{U}(t, \xi) \geq 0 \quad \text { for }(t, \xi) \in[0, \tau] \times \mathbb{R} .
$$

Combining (3.16) and 3.18 , we have

$$
|\widetilde{U}(t, \xi)| \leq U^{+}(t, \xi) \quad \text { for }(t, \xi) \in[0, \tau] \times \mathbb{R} .
$$

Next, when $t \in[\tau, 2 \tau]$, namely, $t-\tau \in[0, \tau]$, based on $(3.19)$, we can similarly prove

$$
\begin{aligned}
& V^{-}(t, \xi)=U^{+}(t, \xi)-\widetilde{U}(t, \xi) \geq 0, \\
& V^{+}(t, \xi)=U^{+}(t, \xi)+\widetilde{U}(t, \xi) \geq 0
\end{aligned}
$$

for $(t, \xi) \in[\tau, 2 \tau] \times \mathbb{R}$, namely,

$$
|\widetilde{U}(t, \xi)| \leq U^{+}(t, \xi) \quad \text { for }(t, \xi) \in[\tau, 2 \tau] \times \mathbb{R} .
$$

Repeating this procedure, we then further prove

$$
|\widetilde{U}(t, \xi)| \leq U^{+}(t, \xi) \quad \text { for }(t, \xi) \in[n \tau,(n+1) \tau] \times \mathbb{R}, n=1,2, \ldots,
$$

which implies

$$
|\widetilde{U}(t, \xi)| \leq U^{+}(t, \xi) \quad \text { for }(t, \xi) \in \mathbb{R}_{+} \times \mathbb{R} .
$$

This completes the proof.

Now we are in a position to establish the decay estimate of the solution $U^{+}$of 3.9 . We first recall some properties of the solutions to the delayed ODE.

Lemma 3.7. 12, Theorem 1] Let $z(t)$ be the solution to the following scalar differential equation with delay

$$
\begin{cases}\frac{d}{d t} z(t)+k_{1} z(t)=k_{2} z(t-\tau), & t \geq 0, \tau>0 \\ z(s)=z_{0}(s), & s \in[-\tau, 0]\end{cases}
$$

Then

$$
z(t)=e^{-k_{1}(t+\tau)} e_{\tau}^{\bar{k}_{2} t} z_{0}(-\tau)+\int_{-\tau}^{0} e^{-k_{1}(t-s)} e_{\tau}^{\bar{k}_{2}(t-\tau-s)}\left[z_{0}^{\prime}(s)+k_{1} z_{0}(s)\right] d s
$$


where

$$
\bar{k}_{2}=k_{2} e^{k_{1} \tau}
$$

and $e_{\tau}^{\bar{k}_{2} t}$ is the so-called delayed exponential function in the form

$$
e_{r}^{\bar{k}_{2} t}= \begin{cases}0, & -\infty<t<-\tau, \\ 1, & -\tau \leq t<0, \\ 1+\bar{k}_{2} \frac{t}{1 !}, & 0 \leq t<\tau, \\ 1+\bar{k}_{2} \frac{t}{1 !}+\bar{k}_{2}^{2} \frac{(t-\tau)^{2}}{2 !}, & \tau \leq t<2 \tau, \\ \vdots & \vdots \\ 1+\bar{k}_{2} \frac{t}{1 !}+\bar{k}_{2}^{2} \frac{(t-\tau)^{2}}{2 !}+\cdots+\bar{k}_{2}^{m \frac{[t-(m-1) \tau]^{m}}{m !},}, & (m-1) \tau \leq t<m \tau, \\ \vdots & \vdots\end{cases}
$$

and $e_{\tau}^{\bar{k}_{2} t}$ is a solution to the following linear homogeneous equation with pure delay

$$
\begin{cases}\frac{d}{d t} z(t)=\bar{k}_{2} z(t-\tau), & t \geq 0, \\ z(s) \equiv 1, & s \in[-\tau, 0] .\end{cases}
$$

Lemma 3.8. 21, Lemma 2.2] Let $k_{1} \geq 0$ and $k_{2} \geq 0$. Then the solution $z(t)$ to (3.20) (or equivalently (3.21)) satisfies

$$
|z(t)| \leq C_{0} e^{-k_{1} t} e_{\tau}^{\bar{k}_{2} t}
$$

where

$$
C_{0}:=e^{-k_{1} \tau}\left|z_{0}(-\tau)\right|+\int_{-\tau}^{0} e^{k_{1} s}\left|z_{0}^{\prime}(s)+k_{1} z_{0}(s)\right| d s,
$$

and the fundamental solution $e_{\tau}^{\bar{k}_{2} t}$ with $\bar{k}_{2}>0$ to 3.22 satisfies

$$
e_{\tau}^{\bar{k}_{2} t} \leq C(1+t)^{-\gamma} e^{\bar{k}_{2} t}
$$

for arbitrary number $\gamma>0$.

Furthermore, when $k_{1} \geq k_{2} \geq 0$, there exists a constant $\epsilon_{1}=\epsilon(\tau)$ with $0<\epsilon<1$ for $\tau>0$, and $\epsilon_{1}=1$ for $\tau=0$, and $\epsilon_{1}=\epsilon(\tau) \rightarrow 0$ as $\tau \rightarrow+\infty$, such that

$$
e^{-k_{1} t} e_{\tau}^{\bar{k}_{2} t} \leq C e^{-\epsilon_{1}\left(k_{1}-k_{2}\right) t}, \quad t>0
$$

and the solution $z(t)$ to 3.20 satisfies

$$
|z(t)| \leq C e^{-\epsilon_{1}\left(k_{1}-k_{2}\right) t}, \quad t>0 .
$$


Taking Fourier's transform to 3.9$)$, and denoting the Fourier's transform of $U^{+}(t, \xi)$ by $\widehat{U}^{+}(t, \eta)$, we obtain

$$
\left\{\begin{array}{l}
\frac{d}{d t} \widehat{U}^{+}(t, \eta)+A(\eta) \widehat{U}^{+}(t, \eta)=B(\eta) \widehat{U}^{+}(t-\tau, \eta), \\
\widehat{U}^{+}(s, \eta)=\widehat{U}_{0}^{+}(s, \eta), \quad s \in[-\tau, 0], \eta \in \mathbb{R}
\end{array}\right.
$$

where

$$
\begin{aligned}
& A(\eta):=c_{1}-d\left(e^{\lambda+\mathbf{i} \eta}+e^{-(\lambda+\mathbf{i} \eta)}\right)+\mathbf{i} c \eta, \\
& B(\eta):=g^{\prime}(0) \sum_{i \in \mathbb{Z}} e^{-\mathbf{i}(i+c \tau) \eta} K(i) e^{-\lambda(i+c \tau)} .
\end{aligned}
$$

By the solution formula 3.21) in Lemma 3.7, the linear time-delayed ordinary differential equation 3.24 can be solved by

$$
\begin{aligned}
\widehat{U}^{+}(t, \eta)= & e^{-A(\eta)(t+\tau)} e_{\tau}^{\bar{B}(\eta) t} \widehat{U}_{0}^{+}(-\tau, \eta) \\
& +\int_{-\tau}^{0} e^{-A(\eta)(t-s)} e_{\tau}^{\bar{B}(\eta)(t-\tau-s)}\left[\partial_{s} \widehat{U}_{0}^{+}(s, \eta)+A(\eta) \widehat{U}_{0}^{+}(s, \eta)\right] d s,
\end{aligned}
$$

where

$$
\bar{B}(\eta):=B(\eta) e^{A(\eta) \tau}
$$

Taking the inverse Fourier transform to 3.26 , we have

$$
\begin{aligned}
U^{+}(t, \xi)= & \frac{1}{2 \pi} \int_{-\infty}^{\infty} e^{\mathbf{i} \xi \eta} e^{-A(\eta)(t+\tau)} e_{\tau}^{\bar{B}(\eta) t} \widehat{U}_{0}^{+}(-\tau, \eta) d \eta \\
& +\frac{1}{2 \pi} \int_{-\tau}^{0} \int_{-\infty}^{\infty} e^{\mathbf{i} \xi \eta} e^{-A(\eta)(t-s)} e_{\tau}^{\bar{B}(\eta)(t-\tau-s)}\left[\partial_{s} \widehat{U}_{0}^{+}(s, \eta)+A(\eta) \widehat{U}_{0}^{+}(s, \eta)\right] d \eta d s
\end{aligned}
$$

We now estimate the decay rate of $U^{+}(t, \xi)$.

Lemma 3.9. Let the initial data $U_{0}^{+}(s, \xi)$ be such that

$$
U_{0}^{+} \in C\left([-\tau, 0] ; W^{1,1}(\mathbb{R})\right) \quad \text { and } \quad \partial_{s} U_{0}^{+} \in L^{1}\left([-\tau, 0] ; L^{1}(\mathbb{R})\right) \text {. }
$$

Then

$$
\left\|U^{+}(t)\right\|_{L^{\infty}(\mathbb{R})} \leq C e^{-\mu_{2} t}
$$

for any $c>\widetilde{c}$ and some $\mu_{2}>0$.

Proof. By Parseval's inequality, it then follows from 3.26 that

$$
\left\|U^{+}(t)\right\|_{L^{\infty}(\mathbb{R})} \leq\left\|\widehat{U}^{+}(t)\right\|_{L^{1}(\mathbb{R})} \leq I_{1}(t)+I_{2}(t),
$$


where

$$
\begin{aligned}
& I_{1}(t):=\int_{-\infty}^{\infty}\left|e^{-A(\eta)(t+\tau)} e_{\tau}^{\bar{B}(\eta) t} \widehat{U}_{0}^{+}(-\tau, \eta)\right| d \eta \\
& I_{2}(t):=\int_{-\tau}^{0} \int_{-\infty}^{\infty}\left|e^{-A(\eta)(t-s)} e_{\tau}^{\bar{B}(\eta)(t-\tau-s)}\left[\partial_{s} \widehat{U}_{0}^{+}(s, \eta)+A(\eta) \widehat{U}_{0}^{+}(s, \eta)\right]\right| d \eta d s .
\end{aligned}
$$

We are going to estimate $I_{i}(t), i=1,2$. By (3.25), one has

$$
\begin{aligned}
\left|e^{-A(\eta)(t+\tau)}\right| & =e^{-c_{1}(t+\tau)}\left|\exp \left((t+\tau) d\left(e^{\lambda+\mathbf{i} \eta}+e^{-(\lambda+\mathbf{i} \eta)}\right)\right)\right| \\
& =e^{-c_{1}(t+\tau)} \exp \left((t+\tau) d\left(e^{\lambda} \cos \eta+e^{-\lambda} \cos \eta\right)\right) \\
& =e^{-c_{1}(t+\tau)} \exp \left((t+\tau) d\left(e^{\lambda}+e^{-\lambda}\right) \cos \eta\right) \\
& =e^{-k_{1}(c)(t+\tau)} e^{-m(\eta)(t+\tau)}
\end{aligned}
$$

where

$$
k_{1}(c):=c_{1}-d\left(e^{\lambda}+e^{-\lambda}\right) \quad \text { and } \quad m(\eta):=(1-\cos \eta) d\left(e^{\lambda}+e^{-\lambda}\right) .
$$

By (3.27) and (3.25), we obtain

$$
|\bar{B}(\eta)|=|B(\eta)|\left|e^{A(\eta) \tau}\right| \leq e^{d\left(e^{\lambda}+e^{-\lambda}\right) \tau} g^{\prime}(0) G(\lambda) e^{k_{1}(c) \tau}=: \bar{k}_{2}(c),
$$

where

$$
\bar{k}_{2}(c):=k_{2}(c) e^{k_{1}(c) \tau} \quad \text { and } \quad k_{2}(c):=e^{d\left(e^{\lambda}+e^{-\lambda}\right) \tau} g^{\prime}(0) G(\lambda) .
$$

Then we get

$$
\left|e_{\tau}^{\bar{B}(\eta) t}\right| \leq e_{\tau}^{|\bar{B}(\eta)| t}=e_{\tau}^{\bar{k}_{2}(c) t}
$$

It is easy to see that

$$
\begin{aligned}
k_{1}(c)-k_{2}(c) & =c \lambda-d\left(e^{\lambda}+e^{-\lambda}-2\right)+1-e^{d\left(e^{\lambda}+e^{-\lambda}\right) \tau} g^{\prime}(0) G(\lambda) \\
& =c \lambda-d\left(e^{\lambda}+e^{-\lambda}-2\right)+1-e^{d\left(e^{\lambda}+e^{-\lambda}\right) \tau} g^{\prime}(0) \sum_{i \in \mathbb{Z}} K(i) e^{-\lambda(i+c \tau)} \\
& \geq c \lambda-d\left(e^{\lambda}+e^{-\lambda}-2\right)+1-e^{d\left(e^{\lambda}+e^{-\lambda}\right) \tau} g^{\prime}(0) \sum_{i \in \mathbb{Z}} K(i) e^{-\lambda i}>0,
\end{aligned}
$$

due to (2.5). Let $\mu_{0}:=k_{1}(c)-k_{2}(c)>0$. Then by 3.23$)$, we have

$$
\begin{aligned}
\left|e^{-A(\eta)(t+\tau)} e_{\tau}^{\bar{B}(\eta) t}\right| & \leq e^{-m(\eta)(t+\tau)} e^{-k_{1}(c)(t+\tau)} e_{\tau}^{\bar{k}_{2}(c) t} \\
& \leq C e^{-\varepsilon_{1}\left[k_{1}(c)-k_{2}(c)\right] t} e^{-m(\eta) t}=C e^{-\varepsilon_{1} \mu_{0} t} e^{-m(\eta) t}
\end{aligned}
$$

Applying (3.29), we derive the optimal estimate for $I_{1}(t)$ :

$$
\begin{aligned}
I_{1}(t) & =\int_{-\infty}^{\infty}\left|e^{-A(\eta)(t+\tau)} e_{r}^{\bar{B}(\eta) t} \widehat{U}_{0}^{+}(-\tau, \eta)\right| d \eta \\
& \leq C e^{-\varepsilon_{1} \mu_{0} t}\left\|\widehat{U}_{0}^{+}(-\tau)\right\|_{L^{\infty}(\mathbb{R})} \int_{-\infty}^{\infty} e^{-m(\eta) t} d \eta \\
& \leq C e^{-\mu_{2} t}\left\|U_{0}^{+}(-\tau)\right\|_{L^{1}(\mathbb{R})},
\end{aligned}
$$


where $\mu_{2}:=\varepsilon_{1} \mu_{0}$.

Similarly, we can estimate $I_{2}(t)$. Note that

$$
\begin{aligned}
\sup _{\eta \in R}\left|A(\eta) \widehat{U}_{0}^{+}(s, \eta)\right| & =\sup _{\eta \in R}\left|\left[c_{1}-d\left(e^{\lambda+\mathbf{i} \eta}+e^{-(\lambda+\mathbf{i} \eta)}\right)+\mathbf{i} c \eta\right] \widehat{U}_{0}^{+}(s, \eta)\right| \\
& \leq C\left\|U_{0}^{+}(s)\right\|_{W^{1,1}(\mathbb{R})} .
\end{aligned}
$$

Thus, we can derive the decay rate for $I_{2}(t)$ as follows:

$$
\begin{aligned}
I_{2}(t) & =\int_{-\tau}^{0} \int_{-\infty}^{\infty}\left|e^{-A(\eta)(t-s)} e_{r}^{\bar{B}(\eta)(t-\tau-s)}\left[\partial_{s} \widehat{U}_{0}^{+}(s, \eta)+A(\eta) \widehat{U}_{0}^{+}(s, \eta)\right]\right| d \eta d s \\
& \leq C \int_{-\tau}^{0} \int_{-\infty}^{\infty} e^{-\varepsilon_{1} \mu_{0}(t-s)} e^{-m(\eta)(t-s)}\left|\left[\partial_{s} \widehat{U}_{0}^{+}(s, \eta)+A(\eta) \widehat{U}_{0}^{+}(s, \eta)\right]\right| d \eta d s \\
& \leq C e^{-\varepsilon_{1} \mu_{0} t} \int_{-\tau}^{0} \int_{-\infty}^{\infty} e^{-m(\eta)(t-s)} \sup \left|\left[\partial_{s} \widehat{U}_{0}^{+}(s, \eta)+A(\eta) \widehat{U}_{0}^{+}(s, \eta)\right]\right| d \eta d s \\
& \leq C e^{-\varepsilon_{1} \mu_{0} t} \int_{-\tau}^{0}\left[\left\|\partial_{s} U_{0}^{+}(s)\right\|_{L^{1}(\mathbb{R})}+\left\|U_{0}^{+}(s)\right\|_{W^{1,1}(\mathbb{R})}\right] d s \\
& \leq C e^{-\mu_{2} t}\left[\left\|\partial_{s} U_{0}^{+}(s)\right\|_{L^{1}\left([-\tau, 0] ; L^{1}(\mathbb{R})\right)}+\left\|U_{0}^{+}(s)\right\|_{\left.L^{1}\left([-\tau, 0] ; W^{1,1}(\mathbb{R})\right)\right] .}\right.
\end{aligned}
$$

Substituting (3.30) and (3.31) to 3.28), we obtain

$$
\left\|U^{+}(t)\right\|_{L^{\infty}(\mathbb{R})} \leq C e^{-\mu_{2} t} \quad \text { for } c>\widetilde{c} .
$$

The proof is complete.

Let us choose $U_{0}^{+}(s, \xi)$ such that $U_{0}^{+} \in C\left([-\tau, 0] ; W^{1,1}(\mathbb{R})\right)$ and $\partial_{s} U_{0}^{+} \in L^{1}([-\tau, 0]$; $\left.L^{1}(\mathbb{R})\right)$, and $U_{0}^{+}(s, \xi) \geq\left|\widetilde{U}_{0}(s, \xi)\right|$ for $(s, \xi) \in[-\tau, 0] \times \mathbb{R}$. Then combining Lemmas 3.6 and 3.9 , we immediately obtain the convergence rate for $\widetilde{U}(t, \xi)$.

Lemma 3.10. When $\widetilde{U}_{0} \in C\left([-\tau, 0] ; W^{1,1}(\mathbb{R})\right)$ and $\partial_{s} \widetilde{U}_{0} \in L^{1}\left([-\tau, 0] ; L^{1}(\mathbb{R})\right)$, then

$$
\|\widetilde{U}(t)\|_{L^{\infty}(\mathbb{R})} \leq C e^{-\mu_{2} t} \quad \text { for } c>\widetilde{c} .
$$

Proposition 3.11. It holds

$$
\sup _{\xi \in\left(-\infty, x_{0}\right]}|U(t, \xi)| \leq C e^{-\mu_{2} t} \quad \text { for } c>\widetilde{c} .
$$

Proof. Notice that $\widetilde{U}(t, \xi)=\sqrt{\omega(\xi)} U\left(t, \xi+x_{0}\right)=e^{-\lambda \xi} U\left(t, \xi+x_{0}\right)$, and $\sqrt{\omega(\xi)}=e^{-\lambda \xi} \geq 1$ for $\xi \in(-\infty, 0]$. Then we obtain

$$
\sup _{\xi \in(-\infty, 0]}\left|U\left(t, \xi+x_{0}\right)\right| \leq\|\widetilde{U}(t)\|_{L^{\infty}(\mathbb{R})} \leq C e^{-\mu_{2} t} \quad \text { for } c>\widetilde{c},
$$

which implies

$$
\sup _{\xi \in\left(-\infty, x_{0}\right]}|U(t, \xi)| \leq C e^{-\mu_{2} t} \quad \text { for } c>\widetilde{c} .
$$

The proof is complete. 


\section{Acknowledgments}

The second author was supported by NSF of China (11861056) and NSF of Gansu Province (18JR3RA093).

\section{References}

[1] M. Aguerrea, C. Gomez and S. Trofimchuk, On uniqueness of semi-wavefronts, Math. Ann. 354 (2012), no. 1, 73-109.

[2] X. Bao, W. Shen and Z. Shen, Spreading speeds and traveling waves for space-time periodic nonlocal dispersal cooperative systems, Commun. Pure Appl. Anal. 18 (2019), no. $1,361-396$.

[3] X. Chen and J.-S. Guo, Existence and asymptotic stability of traveling waves of discrete quasilinear monostable equations, J. Differential Equations 184 (2002), no. 2, $549-569$.

[4] I.-L. Chern, M. Mei, X. Yang and Q. Zhang, Stability of non-monotone critical traveling waves for reaction-diffusion equations with time-delay, J. Differential Equations 259 (2015), no. 4, 1503-1541.

[5] J. Fang, J. Wei and X.-Q. Zhao, Spreading speeds and travelling waves for nonmonotone time-delayed lattice equations, Proc. R. Soc. Lond. Ser. A Math. Phys. Eng. Sci. 466 (2010), no. 2119, 1919-1934.

[6] _ Uniqueness of traveling waves for nonlocal lattice equations, Proc. Amer. Math. Soc. 139 (2011), no. 4, 1361-1373.

[7] J.-S. Guo and Y.-C. Lin, Traveling wave solution for a lattice dynamical system with convolution type nonlinearity, Discrete Contin. Dyn. Syst. 32 (2012), no. 1, 101-124.

[8] _ Entire solutions for a discrete diffusive equation with bistable convolution type nonlinearity, Osaka J. Math. 50 (2013), no. 3, 607-629.

[9] S. Guo and J. Zimmer, Stability of travelling wavefronts in discrete reaction-diffusion equations with nonlocal delay effects, Nonlinearity 28 (2015), no. 2, 463-492.

[10] C. Hu and B. Li, Spatial dynamics for lattice differential equations with a shifting habitat, J. Differential Equations 259 (2015), no. 5, 1967-1989.

[11] R. Huang, M. Mei, K. Zhang and Q. Zhang, Asymptotic stability of non-monotone traveling waves for time-delayed nonlocal dispersion equations, Discrete Contin. Dyn. Syst. 36 (2016), no. 3, 1331-1353. 
[12] D. Ya. Khusainov, A. F. Ivanov and I. V. Kovarzh, Solution of one heat equation with delay, Nonlinear Oscil. 12 (2009), no. 2, 260-282.

[13] C.-K. Lin, C.-T. Lin, Y. Lin and M. Mei, Exponential stability of nonmonotone traveling waves for Nicholson's blowflies equation, SIAM J. Math. Anal. 46 (2014), no. 2, 1053-1084.

[14] S. Ma, P. Weng and X. Zou, Asymptotic speed of propagation and traveling wavefronts in a non-local delayed lattice differential equation, Nonlinear Anal. 65 (2006), no. 10, 1858-1890.

[15] S. Ma and X. Zou, Existence, uniqueness and stability of travelling waves in a discrete reaction-diffusion monostable equation with delay, J. Differential Equations 217 (2005), no. 1, 54-87.

[16] _ Propagation and its failure in a lattice delayed differential equation with global interaction, J. Differential Equations 212 (2005), no. 1, 129-190.

[17] M. Mei, C.-K. Lin, C.-T. Lin and J. W.-H. So, Traveling wavefronts for time-delayed reaction-diffusion equation I: Local nonlinearity, J. Differential Equations 247 (2009), no. $2,495-510$.

[18] _ , Traveling wavefronts for time-delayed reaction-diffusion equation II: Nonlocal nonlinearity, J. Differential Equations 247 (2009), no. 2, 511-529.

[19] M. Mei and J. W.-H. So, Stability of strong travelling waves for a non-local timedelayed reaction-diffusion equation, Proc. Roy. Soc. Edinburgh Sect. A 138 (2008), no. $3,551-568$.

[20] M. Mei, J. W.-H. So, M. Y. Li and S. S. P. Shen, Asymptotic stability of travelling waves for Nicholson's blowflies equation with diffusion, Proc. Roy. Soc. Edinburgh Sect. A 134 (2004), no. 3, 579-594.

[21] M. Mei and Y. Wang, Remark on stability of traveling waves for nonlocal Fisher-KPP equations, Int. J. Numer. Anal. Model. Ser. B 2 (2011), no. 4, 379-401.

[22] M. Mei, K. Zhang and Q. Zhang, Global stability of critical traveling waves with oscillations for time-delayed reaction-diffusion equations, Int. J. Numer. Anal. Model. 16 (2019), no. 3, 375-397.

[23] G. Tian and G.-B. Zhang, Stability of traveling wavefronts for a discrete diffusive Lotka-Volterra competition system, J. Math. Anal. Appl. 447 (2017), no. 1, 222-242. 
[24] G. Tian, G. Zhang and Z. Yang, Stability of nonmonotone critical traveling waves for spatially discrete reaction-diffusion equations with time delay, Turkish J. Math. 41 (2017), no. 3, 655-680.

[25] P. Weng, H. Huang and J. Wu, Asymptotic speed of propagation of wave fronts in a lattice delay differential equation with global interaction, IMA J. Appl. Math. 68 (2003), no. 4, 409-439.

[26] S.-L. Wu, H.-Q. Zhao and S.-Y. Liu, Asymptotic stability of traveling waves for delayed reaction-diffusion equations with crossing-monostability, Z. Angew. Math. Phys. 62 (2011), no. 3, 377-397.

[27] Z. Xu and J. Ma, Monotonicity, asymptotics and uniqueness of travelling wave solution of a non-local delayed lattice dynamical system, Discrete Contin. Dyn. Syst. 35 (2015), no. 10, 5107-5131.

[28] Z. Yang and G. Zhang, Stability of non-monotone traveling waves for a discrete diffusion equation with monostable convolution type nonlinearity, Sci. China Math. 61 (2018), no. 10, 1789-1806.

[29] Z.-X. Yang, G.-B. Zhang, G. Tian and Z. Feng, Stability of non-monotone non-critical traveling waves in discrete reaction-diffusion equations with time delay, Discrete Contin. Dyn. Syst. Ser. S 10 (2017), no. 3, 581-603.

[30] Z.-X. Yu, Uniqueness of critical traveling waves for nonlocal lattice equations with delays, Proc. Amer. Math. Soc. 140 (2012), no. 11, 3853-3859.

[31] Z. Yu, F. Xu and W. Zhang, Stability of invasion traveling waves for a competition system with nonlocal dispersals, Appl. Anal. 96 (2017), no. 7, 1107-1125.

[32] G.-B. Zhang and R. Ma, Spreading speeds and traveling waves for a nonlocal dispersal equation with convolution-type crossing-monostable nonlinearity, Z. Angew. Math. Phys. 65 (2014), no. 5, 819-844.

Tao Su and Guo-Bao Zhang

College of Mathematics and Statistics, Northwest Normal University, Lanzhou, Gansu 730070, China

E-mail address: 970913788@qq.com, zhanggb2011@nwnu.edu.cn 